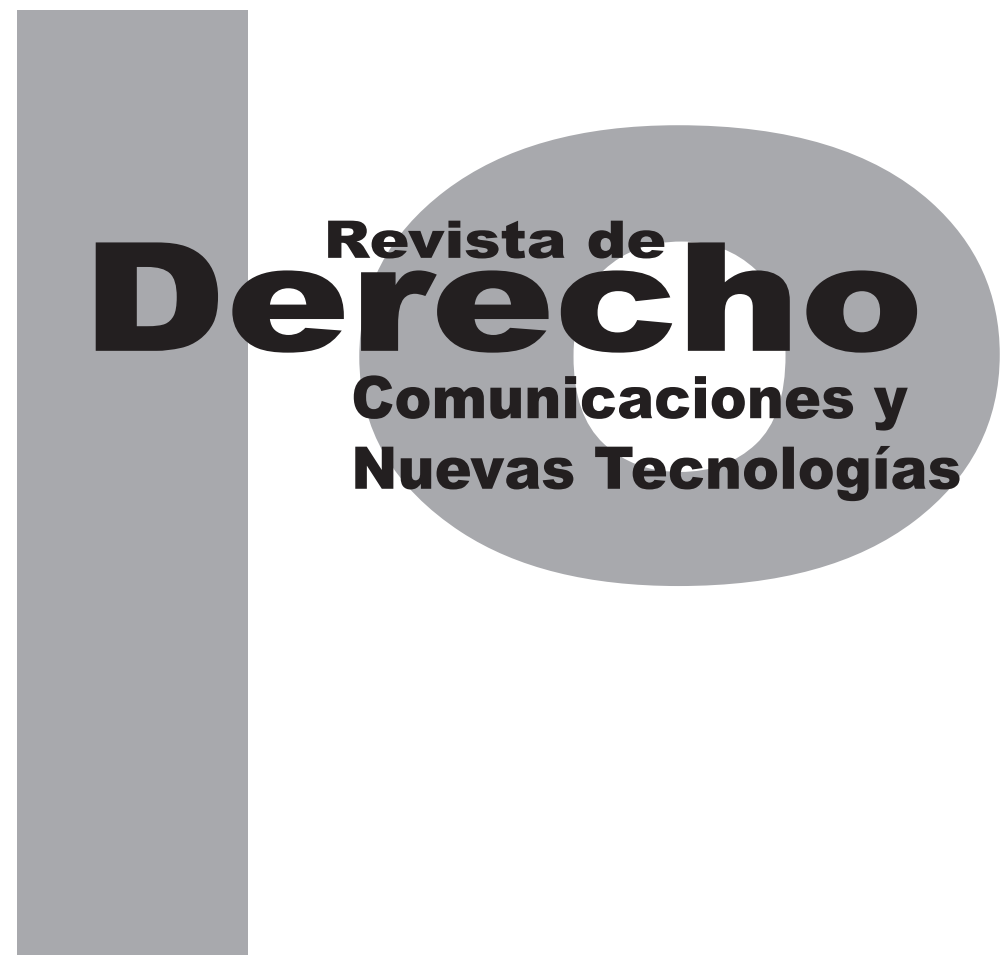

\title{
LAS COMUNIDADES VIRTUALES Y SU IMPORTANCIA PARA LA INTEGRACIÓN DE LOS COLOMBIANOS EN ARGENTINA
}

\author{
CÉsar Guauque TORRES
}

Universidad de los Andes

Facultad de Derecho

Rev. derecho comun. nuevas tecnol.

No. 14, julio - diciembre de 2015. ISSN 1909-7786 


\title{
Las comunidades virtuales y su importancia para la integración de los colombianos en Argentina
}

\section{Resumen}

Este trabajo desarrolla un acercamiento al concepto de comunidad virtual a partir de una dimensión teórica, procurando confrontar los elementos y principales cuestionamientos sobre esta noción en las asociaciones de colombianos establecidas en Facebook, en las ciudades de Bahía Blanca, La Plata y Buenos Aires (Argentina). El objetivo es la comprensión de la relación entre los entornos offline y las posibilidades que para la integración de sus miembros tienen estas formas de agregación e interacción social-virtual. Tomando como método la etnografía virtual se realiza la observación participante sobre el espacio de intertextualidad que permite señalar, con base en la percepción de algunos de los participantes en este tipo de agrupaciones, cuáles son las motivaciones para el ingreso, los sentidos que asume la pertenencia a estos grupos y la influencia del contexto de la vida offline, respecto de las posibilidades que tienen estas asociaciones para la integración de sus miembros.

Palabras clave: redes sociales, comunidades virtuales, etnografía virtual, Facebook, colombianos en Argentina.

\section{Virtual communities and their importance for integration of colombians in Argentina}

\begin{abstract}
This paper develops an approach to the concept of "virtual community" from a theoretical dimension, in that it attempts to confront the elements and main questions of this notion in the context of relationships established among Colombians on Facebook, within the cities of Bahia Blanca, La Plata and Buenos Aires (Argentina). The goal is to understand the relationship between offline environments and the possibilities that these types of gatherings and social-virtual interactions can provide to its members. Using virtual ethnography as a method of participatory observation of inter-textuality allows for the identification, based on the perception of some of the participants in such groups, of the motivations for joining, the assumed sense of belonging to these groups and the influence of their offline lives, with respect to the possibilities that these groups have for the inclusion of its members.
\end{abstract}

Keywords: social networks, virtual communities, virtual ethnography, Facebook, Colombians in Argentina.

\section{As comunidades virtuais e sua importância para a integração dos colombianos na Argentina Resumo}

Este trabalho desenvolve uma aproximação ao conceito de comunidade virtual a partir de uma dimensão teórica, procurando confrontar os elementos e principais questionamentos sobre esta noção nas associações de colombianos estabelecidas no Facebook, nas cidades de Bahía Blanca, La Plata e Buenos Aires (Argentina). O objetivo é a compreensão da relação entre os entornos offline e as possibilidades que para a integração de seus membros têm estas formas de agregação e interação social-virtual. Tomando como método a etnografia virtual se realiza a observação participante sobre o espaço de intertextualidade que permite mostrar, com base na percepção de alguns dos participantes neste tipo de agrupações, quais são as motivações para o ingresso, os sentidos que assume a pertença a estes grupos e a influência do contexto da vida offline, respeito das possibilidades que têm estas associações para a integração de seus membros.

Palavras-chave: redes sociais, comunidades virtuais, etnografia virtual, Facebook, colombianos na Argentina. 


\title{
Las comunidades virtuales y su importancia para la integración de los colombianos en Argentina*
}

\author{
César Guauque Torres**
}

\section{SUMARIO}

Introducción - I. LAS COMUNIDADES VIRTUALES: ORÍGENES DEL CONCEPTO, CRÍTICAS Y PROYECCIÓN - II. ETNOGRAFÍA VIRTUAL. ESTRATEGIA, MATERIALES, TÉCNICAS Y OPERACIONALIZACIÓN - A. Operacionalización: hipótesis, variables e indicadores - III. USO Y APROPIACIÓN DE LA PLATAFORMA DE FACEBOOK POR LAS AGRUPACIONES DE COLOMBIANOS EN LAS CIUDADES DE BAHÍA BLANCA, LA PLATA Y BUENOS AIRES (ARGENTINA) - A. Las motivaciones para el ingreso a las asociaciones en Facebook de colombianos en Argentina - B. Sentidos que asume la participación en las agrupaciones - C. Observación de los tipos de usos y formas de apropiación - IV. DESDE LA COMUNICABILIDAD VIRTUAL HACIA LA INTEGRACIÓN OFFLINE - V. A MODO DE CONCLUSIÓN: UNA CRÍTICA - Referencias.

* Cómo citar este artículo: Guauque Torres, C. (Diciembre, 2015). Las comunidades virtuales y su importancia para la integración de los colombianos en Argentina. Revista de Derecho, Comunicaciones y Nuevas Tecnologías, 14. Universidad de los Andes (Colombia).

** Abogado, Universidad Pedagógica y Tecnológica de Colombia; politólogo y especialista en Filosofía Política, Universidad Nacional de Colombia; magíster en Filosofía del Derecho y Teoría jurídica, Universidad Libre de Colombia; cursa actualmente estudios de Doctorado en Ciencias Sociales en la Universidad Nacional de La Plata (Argentina). Profesor Universidad Industrial de Santander; Universidad Cooperativa de Colombia, sede Bucaramanga; profesor e investigador Universidad Libre seccional Socorro. Correo: cesarguauque@gmail.com 
Introducción

El presente trabajo toma como punto de partida el replanteamiento del concepto de comunidad, desde su formulación tradicional muy centrada en la fortaleza de los vínculos de sujeción e identificación con una conciencia colectiva anclada en tradiciones y contextos culturales muy estáticos, hacia una comprensión más amplia en la cual se incorporen las formas de agregación e interacción social que han tenido lugar a partir del uso y apropiación social de las nuevas tecnologías de la información y la comunicación, en particular la Internet.

La participación en estas formas de agregación e interacción social-virtual pone en el centro de la cuestión la problemática diferenciación real/ virtual, en primer lugar -pero no exclusivamente-, desde la ambigua dislocación que hace posible la presencia simultánea del ser en los espacios online y offline. La posibilidad de recurrir al concepto de comunidad como categoría-metáfora para la comprensión de este fenómeno, pasa entonces a través de la consideración de dos escenarios posibles: por una parte, el que toma a la comunicación como un fin en sí mismo de la agrupación que en el propio espacio virtual de intertextualidad configura símbolos, valores y prácticas vinculantes; o el que tiene en la interacción online solo un medio para profundizar los vínculos de una agrupación offline existente o al menos en formación.

En ese sentido, es importante poder plantear un diálogo entre la formulación conceptual y algunas experiencias actuales de estas nuevas formas de agregación e interacción que han sido o pueden llegar a ser categorizadas desde esta perspectiva. El estudio de la relación entre la presencia online y offline adquiere entonces gran importancia para poder analizar, en cada caso, las condiciones dentro de las cuales estas agrupaciones desarrollan su actividad, pudiendo diferenciarse formaciones con un carácter plenamente translocalizado, como por ejemplo, un grupo de fanáticos de la banda inglesa Radiohead y otro tipo de agregaciones en las cuales sí existe alguna referencia al medio desde el cual despliegan su presencia virtual. En ocasiones esta relación constituye la razón misma del vínculo, como ocurre con las asociaciones de inmigrantes colombianos establecidos en algunas ciudades de la Argentina.

Buenos Aires, pero también ciudades como Córdoba, La Plata y Bahía Blanca han sido receptoras de la actual ola de inmigrantes colombianos que tienen como una de las motivaciones principales la accesibilidad del sistema de educación superior a nivel de grado y posgrados: la posibilidad de becas, los costos y la calidad de la educación son factores muy atractivos en relación con las condiciones actuales en Colombia. Las facilidades que ofrece la política de inmigración argentina son un factor igualmente importante para propiciar este fenómeno.

En cada una de estas ciudades se han venido formando agrupaciones en la plataforma de Facebook, que en su mayoría tienen como propósito principal -señalado expresamente- el generar espacios de encuentro e integración de la comunidad colombiana. Solo en referencia a 
la ciudad de Buenos Aires existen más de cinco grupos, La Plata y Bahía Blanca cuentan con uno principal, todos procuran brindar un espacio para la comunicación e interacción no solo entre quienes comparten la nacionalidad de origen sino también algún tipo de vínculo o afecto con los colombianos en Argentina.

Con este marco de referencia, el presente trabajo se propone indagar sobre la relevancia que los miembros de las agrupaciones en Facebook de colombianos residentes en las ciudades de Bahía Blanca, La Plata y Buenos Aires le atribuyen a estas formas de asociación e interacción social virtual para su integración, de acuerdo con sus condiciones de vida y experiencias en cada una de estas ciudades.

El objetivo de esta indagación es la comprensión de la relación entre los entornos offline, y las posibilidades que para la integración de sus miembros tienen estas formas de agregación e interacción social-virtual. El sustento de este trabajo es la observación de las prácticas de uso y apropiación de la plataforma de Facebook por parte de las agrupaciones de colombianos establecidas en las ciudades mencionadas.

Para el desarrollo de este objetivo presento, en primera instancia, unas consideraciones introductorias sobre la fundamentación del concepto de comunidad virtual, señalando sus principales rasgos y las críticas planteadas sobre su posibilidad, vigencia y proyección. En una segunda parte relaciono los resultados de la aproximación exploratoria a estas formas de agregación social-virtual de colombianos en la República
Argentina, para lo cual realizo una triangulación sobre la población objetivo de este estudio entre las agrupaciones establecidas en la plataforma de Facebook, en las ciudades de Bahía Blanca, La Plata y Buenos Aires. Al interior de cada uno de estos espacios se indagó, a través de la práctica de la etnografía virtual, ${ }^{1}$ sobre las motivaciones para el ingreso, los sentidos que asume la pertenencia a estos grupos y la influencia del contexto de la vida offline, respecto de las posibilidades que tienen estas asociaciones para la integración de sus miembros. ${ }^{2}$

En este punto es preciso señalar que el interés en el tema surge de mi propia condición de inmigrante colombiano en Argentina y el haber tenido la posibilidad de compartir e interactuar con los compatriotas residentes en estas tres ciudades. La motivación de emprender un trabajo de indagación reflexiva sobre estos espacios surge, en parte, de las aproximaciones anteriores al concepto de comunidad desde la filosofía política, y fundamentalmente de las investigaciones y análisis sobre las nuevas tecnologías de la información y la comunicación realizadas en el Seminario Tıc en Perspectiva Política, desarrollado en el marco del Doctorado en Ciencias So-

1 Con fundamento en Christine Hine, la etnografía virtual se entiende como una exploración por medio de la experiencia interactiva y comprometida de la conectividad, estudiando relaciones sociales y formaciones colectivas mediadas por las nuevas tecnologías de la información y la comunicación. Para este trabajo tuvo como actividades específicas la realización de algunas entrevistas semiestructuradas, el desarrollo de un cuestionario a manera de encuesta, actividades enmarcadas dentro de la observación participante planificada.

2 Dentro de esta variable pueden incorporarse aspectos tales como el género, la procedencia, los roles, las posiciones de sujeto, las trayectorias y tramas argumentativas de los propios participantes. Sin embargo, y dado el carácter simplemente exploratorio de este trabajo, solo me centraré en la importancia del contexto local respecto de la participación de los miembros en este tipo de espacios online. 
ciales de la Universidad Nacional de La Plata. La oportunidad de articular a través de una práctica como la etnografía virtual estos dos ámbitos: la experiencia de vida y la formación académica, me parece, en este momento, muy importante y productiva no solo en lo personal, sino también por la expectativa de poder contribuir en los espacios de autorreflexividad de las agrupaciones estudiadas.

\section{LAS COMUNIDADES VIRTUALES: ORÍGENES DEL CONCEPTO, CRÍTICAS Y PROYECCIÓN}

La recuperación del concepto de comunidad como referente del vínculo social tiene lugar a partir de las críticas respecto del nexo orgánicofuncional que en la modernidad colocó como contenido de la conciencia colectiva el culto al individuo. Desde la filosofía política se recurre al imaginario de la polis griega como fundamento ontológico y teleológico para sustentar la existencia de formas de vida que sirven de marco de significación y criterio de orientación para la acción del hombre como ser social capaz de una existencia política (Agamben, 1997). En algunos casos, esta recuperación toma como eje central la crítica del universalismo abstracto del proyecto de la modernidad ilustrada y la fundamentación liberal del individuo (Maclntyre, 1987, pág. 322), operando una justificación del retorno hacia formas de vida antimodernas en las cuales se puedan restablecer tradiciones y vínculos morales que operen como un sistema de creencias y sentimientos comunes.
No obstante, el desarrollo de las nuevas tecnologías de la información y la comunicación, a partir de las últimas dos décadas del siglo pasado, fue abonando un terreno muy fértil para el surgimiento de otras formas de interacción y asociación social que no tienen como principio para su existencia el sentido de pertenencia o adscripción a una conciencia colectiva fijada a partir de tradiciones o fundamentos comprensivos. De acuerdo con Jesús Martín Barbero:

Las tecnologías de la información y la comunicación se constituyen en enclaves de condensación e interacción de mediaciones sociales, conflictos simbólicos e intereses económicos y políticos al tiempo que son el espacio para la emergencia de nuevos sujetos, nuevas relaciones, nuevas formas de crear, compartir y narrar. (Citado por García y Uscátegui, 2011, pág. 52).

La reestructuración del espacio-tiempo generada por la expansión de los medios masivos de comunicación en el orden simbólico-cultural y por la vía de la profundización de los intercambios y flujos migratorios, estimula y redimensiona el trabajo inmaterial afectivo de interacción y contacto humano (Hardt y Negri, 2000, pág. 254), como trabajo de la imaginación (Appadurai, 2001).

De acuerdo con Appadurai (2001), el trabajo de la imaginación es posible por su desprendimiento tanto del espacio expresivo inmediato (próximo, directamente experimentado) como del orden trascendente (ligado al arte, mito o ritual) y su proyección hacia la acción/expresión dentro de la praxis cotidiana como un hecho social y colectivo. Se produce una nueva subjetividad que 
discurre entre su presencia virtual y física en formas de vinculación por cuya intermediación es posible hacer el tránsito de la imaginación compartida a la acción colectiva. Tales formas son cada vez más translocales, desvinculadas del imaginario de los Estados-nación, configuradas por el momento solo como esferas públicas en diáspora pero obligadas a desarrollar procesos de autoestructuración para poder sobrevivir en la disputa sobre la constitución de los imaginarios sociales.

La actualización de esa nueva subjetividad en las prácticas cotidianas debe permitir simultáneamente el reconocimiento y la diferenciación; es allí donde el carácter heurístico de la dimensión cultural cobra relevancia para la recreación de los imaginarios virtuales en el uso y disposición por parte de un colectivo de identificación, que lo expresa en términos acordes con sus particularidades y contextos de significación. Lo local sería ese exceso de la forma como esos múltiples flujos y procesos de lo global actúan sobre lo local: una búsqueda de lo distintivo y lo propio dentro de un permanente trabajo de identificación, de sedimentación de las particularidades que asume el cruce de esos flujos en un contexto determinado.

Sobre la base de esas esferas públicas en diáspora, configuradas en el orden de la materialidad espectral principalmente en estado potencial, es que se producen las formas de agregación social que han procurado ser comprendidas conforme a la figura-metáfora de la comunidad. La mayoría de los especialistas atribuyen a Rheingold (1993) las definiciones fun- dacionales de esta perspectiva. La comunidad virtual es comprendida como "una red electrónica autodefinida de comunicación interactiva, organizada en torno a un interés o propósito compartido (en donde), a veces, la comunicación se convierte en sí misma en la meta" (Rheingold, citado por Castells, 2009, pág. 395).

Como elementos importantes de la comunidad virtual destacamos, entre otros: los imaginarios sociales sobre los que se sustenta (la sociedad de la información, las redes sociales, Internet como espacio y como cultura); su carácter público y cada vez más accesible; las prácticas de reconocimiento mutuo que propicia (Burch, 2011); las posibilidades de interacción "produciendo cierta convergencia entre el orden comunicativo y la participación política no convencional" (Sádaba, 2011, pág. 3); el cuestionamiento del liderazgo y la representación como forma de intervención en el mundo social y político (Valderrama, 2008, pág. 97); la atenuación —en su interior- de los condicionantes de inclusión/ exclusión materiales; la posibilidad de aparecer permaneciendo parcial o totalmente "incógnito"; y, sobre todo, su carácter real, "en la medida en que produce efectos reales en la inter-subjetividad colectiva y en la formación de la opinión pública" (Winocur, 2005, pág. 86).

No obstante estos elementos novedosos, se plantean una serie de críticas respecto de la posibilidad de comprender este tipo de formaciones sociales con fundamento en el concepto de comunidad, en primera instancia, por la evidente fragilidad del vínculo en relación con las nociones de compromiso/responsabilidad/sen- 
tido de pertenencia a un colectivo, propias de la concepción tradicional del término; prácticas como la multipresencia, la facilidad de entrar y salir para los merodeadores o fisgones, la posibilidad de explotar una personalidad diferente a la que se tiene en la vida offline (Hine, 2004, pág. 32), la evasión; y sobre todo la dislocación profunda que permite "estar sin estar" en la comodidad de la presencia espectral, contribuyen a erosionar la factibilidad de aplicación de esta categoría-metáfora.

Es posible señalar, conforme a estos cuestionamientos, que el elemento más problematizado respecto de la participación en estas formas de agregación e interacción social-virtual es la idea de autenticidad, más aún, la remisión a la noción de identidad como referente de esa pretendida correspondencia entre lo expresado en la presencia online y los elementos que configuran la vida offline.

La referencia a la identidad como fundamento de la comunidad plantea, respecto del tipo de formaciones sociales estudiadas, varios problemas adicionales a considerar, más allá del cuestionamiento mismo del esencialismo de la perspectiva identitaria: la configuración de la identidad anterior a la participación mediada, la posibilidad del medio como articulador de identidad en el espacio de intertextualidad y la producción de una identidad a posteriori atravesada por los rasgos constituidos en la interacción entre una y otra dimensión.

Respecto de la existencia en ese ambiguo lugar de indiferenciación entre la presencia virtual y física, y tomando distancia de la comprensión tecnooptimista que tiende a sobredimensionar las posibilidades inherentes al medio en sí mismo, varios autores reivindican el momento y las condiciones de reterritorialización como constitutivas de este tipo de formas sociales. En este sentido, la crítica del enfoque que le atribuía a las comunicaciones mediadas por ordenador (смо) la propiedad de reducción de los signos sociales atribuibles al contexto (Hine, 2004, pág. 27), permite señalar que el grado de cohesión de las formaciones sociales en espacios online se encuentra estrechamente vinculado con factores asociados a la existencia física en un espacio determinado, tales como el género, la raza, el nivel educativo, el acceso a bienes y servicios, la garantía de los derechos básicos, los procesos de subjetivación, de asunción de roles, las trayectorias de los sujetos, así como el territorio donde se habita y sus imaginarios.

Enmarcando las críticas sobre el concepto de comunidad virtual en el orden más amplio de los cuestionamientos frente a las nuevas formas de vinculación social que han pretendido ser explicadas desde este paradigma (comunidades postmodernas), vemos cómo este nuevo tipo de agrupaciones tiene un carácter más o menos transitorio y fluido en el que sus miembros encuentran integración gracias a su afinidad expresiva y afectiva, son tanto comunidades emocionales como comunidades morales que involucran formas de acción afectiva y de valor-racionales (Ayora Díaz, 2003); estos grupos de sujetos reconocen que comparten una cierta estética de la praxis social, que se puede manifestar en sus gustos, en el consumo, 
así como en cualquier otra forma de expresión estética.

Para autores como Bauman (2001), estos "simulacros de comunidad" proporcionan una identidad colectiva, un estilo de vida y un contexto para la interpretación; también se pueden comprender como un modelo de colectividad en el cual se presenta una vía de escape para el miedo y la furia acumulada, espacio de agregación de múltiples singularidades, copado por lo privado que no puede ser tomado como nuestro (común), lugar de la evasión cómplice y la catarsis colectiva para esa multiplicidad de individuos encerrados en su propia soledad. Tales formas de sociabilidad no constituyen un referente de sentido independiente de las voluntades individuales (polisémicas) que las conforman; serían por lo tanto inviables, precisamente porque la tarea de hacer frente a la desprotección está privatizada o más grave aún, está individualizada (privatización de los miedos).

Una vez presentado el marco general, algunos rasgos principales y los cuestionamientos sobre la viabilidad del concepto, corresponde a esta altura entrar de lleno en la confrontación de las herramientas teórico-analíticas en el caso objeto de estudio. Para estos efectos es preciso relacionar la estrategia de investigación a seguir, el tipo de material sobre el cual versará este estudio, la forma de operacionalización del problema y las técnicas de recolección de la información a utilizar.

\section{ETNOGRAFÍA VIRTUAL. ESTRATEGIA, MATERIALES, TÉCNICAS Y OPERACIONALIZACIÓN}

La práctica de la etnografía virtual busca entrar en la experiencia compartida de producción de sentidos comunes al interior de la formación social objeto de estudio, verificar lo que la gente hace con la tecnología, qué se hace, por qué y en qué términos (Hine, 2004, pág. 33). Si bien parto de reconocer su carácter parcial, flexible y adaptativo (Hine, 2004, págs. 81-82), planteo un orden estratégico para el abordaje del objeto de estudio: inicio con una relación breve y necesaria de los antecedentes y presupuestos para la intervención participativa en los escenarios, continúo con las entrevistas a informantes claves para la interacción con el grupo, y desarrollo luego una invitación más amplia a la participación dentro de la investigación a través de la práctica de un cuestionario a modo de muestreo no representativo, todo ello enmarcado dentro de la observación participante planificada en el medio estudiado.

Una vez definida la estrategia es pertinente señalar que el material sobre el cual versará este abordaje son los textos compartidos por los participantes a través de la plataforma. La webgrafía como forma de textografía ${ }^{3}$ sería una forma parcial de etnografía por encontrarse centrada en los textos y su circulación, más que en las interacciones que se producen a partir de es-

3 La textografía intenta combinar el análisis textual interpretando su relación con otros textos y las circunstancias de producción de sus autores (Swales, citado por Hine, 2004, pág. 67). 
tos textos. No obstante, para el caso en estudio se trata de asociar la interacción como intertextualidad con el contexto de producción de estas manifestaciones. De acuerdo con Hine (2004, pág. 48), en función de esta relación:

En vez de poseer cualidades inherentes, el texto de la tecnología "hace posibles" ciertas lecturas que son interpretadas/usadas según el contexto, lo cual no significa que sean los contextos los que tienen cualidades inherentes pues las características relevantes de cada contexto también se producen en los momentos de interacción con la tecnología.

El carácter intertextual de las cмо le confiere una connotación simplemente hermenéutica al estudio de estas formas de comprensión de la realidad que las personas expresan a través de los espacios online. Los textos, en este caso, expresan uno o varios aspectos de la pluridimensionalidad en la que se encuentra una persona; como juicios del orden dialéctico no pueden ser sometidos a una prueba de autenticidad, sino simplemente ser considerados como tópicos cuya valoración se da en términos de lo simplemente opinable o verosímil y no de proposiciones primeras o verdaderas.

En relación con el carácter multisituado de la etnografía en espacios online es preciso aclarar que, para los casos que se va a entrar a observar, si bien se explora a través de "la experiencia interactiva y comprometida de la conectividad, en donde el espacio se entiende más en función de la conectividad que de la localización" (Hine, 2004, pág. 77), no es posible la ruptura plena con la espacialidad física, por cuanto la existen- cia de la agrupación virtual tiene una explícita y fundamental referencia a una locación geográfica. Los procesos culturales, en este caso, se producen en la compleja interacción entre el lugar físico y la presencia virtual.

\section{A. Operacionalización: hipótesis, variables $e$ indicadores}

Tomando como punto de referencia la distinción planteada por Hine (2004) entre la comprensión de Internet como cultura y como artefacto cultural, ${ }^{4}$ en relación con el uso y apropiación de la plataforma de Facebook por parte de las asociaciones de colombianos en las ciudades de Bahía Blanca, La Plata y Buenos Aires, en este trabajo sostengo que dadas las condiciones del contexto offline presentes en cada uno de los casos, se observan dos tipos de formas de agregación e interacción: una que toma a la comunicación como un fin en sí mismo de la agrupación que en el propio espacio virtual de intertextualidad configura símbolos, valores y prácticas vinculantes, pero a través de una forma de sociabilidad débil ${ }^{5}$ - sería el caso de los grupos de las ciudades de La Plata y Buenos Aires-, y otra que tiene en la interacción online solo un medio para profundizar los vínculos de

4

La primera sostiene que Internet representa un lugar donde se gesta una cultura: el ciberespacio. La segunda define al Internet como un artefacto cultural (Woolgar, 1996), un producto de la cultura, en fin: una tecnología que ha sido generada por personas concretas, con objetivos y prioridades contextualmente situados y definidos y, también, conformada por los modos en que ha sido comercializada, enseñada y utilizada (Hine, 2004, pág. 19).

5 Sin llegar a ser simplemente una colección de contactos, se consideran formados por "vínculos débiles" las formas de agregación caracterizadas por el contacto poco frecuente, la falta de cercanía emocional y una corta o inexistente historia de favores recíprocos (Sádaba, 2011, pág. 2). 
una agrupación offline existente fundada en vínculos de sociabilidad fuertes - sería el caso de la ciudad de Bahía Blanca-.

Sobre el escenario de intertextualidad entro a considerar una serie de variables, con fundamento en las cuales se pueda establecer la influencia del contexto offline sobre estas formas de agregación virtual:

(1) El uso con fines particulares del medio colectivo (avisos comerciales, la creación de eventos, compartir información, el contacto y la conectividad). Acá el uso de la plataforma tiene un interés (para) de carácter particular y la comunicación no es el fin mismo de la agrupación.

(2) El uso colectivo para las posibilidades de integración offline (desde la programación de encuentros casuales recreativos o de entretenimiento, reuniones de grupos o "parches" con algún objetivo -cultural, social, religioso, político-, actividades de intervención y acción política, organización como colectividad colombiana. Acá el uso de la plataforma tiene un interés (para) de carácter colectivo y la comunicación no es el fin mismo de la agrupación.

(3) La apropiación expresiva para las manifestaciones de autoafirmación simbólica del grupo, a través de valores, discursos, tópicos. En este caso la comunicación es tomada como el espacio en sí de la comunidad.

(4) La apropiación para desarrollar formas de asistencia y apoyo (recomendaciones, con- sejos, ayuda). Aquí también la comunicación es tomada como el espacio en sí de la comunidad, aun cuando la interacción se proyecta en algunas ocasiones hacia el mundo offline.

(5) Por último, la apropiación para la problematización frente a tensiones, temas de coyuntura o de interés para los participantes tanto sobre su condición de migrante como sobre la situación de Colombia.

Con fundamento en estas variables a observar (los tipos de usos y apropiaciones) dentro del espacio de intertextualidad provisto por la plataforma de Facebook a las agrupaciones objetivo de este estudio, entro a esclarecer la relación entre el contexto offline y la forma que adquiere la interacción y participación en la agrupación, sobre la base de las dos posibilidades contempladas en la hipótesis. Como indicadores de tal relación se establecen los siguientes:

(1) Referencias a integración, encuentros, entretenimiento, vida social: iniciativas individuales, como grupo(s), como colectividad.

(2) Referencia, intervención y participación en eventos y acontecimientos locales: iniciativas individuales, como grupo(s), como colectividad.

(3) Referencias a reuniones de organización como colectividad. 
III. USO Y APROPIACIÓN DE LA PLATAFORMA DE FACEBOOK POR LAS AGRUPACIONES DE COLOMBIANOS EN LAS CIUDADES DE BAHÍA BLANCA, LA PLATA Y BUENOS AIRES (ARGENTINA)

El interés en este trabajo de exploración tiene que ver, en primera instancia, con mi propia condición de inmigrante colombiano en la Argentina y, en segundo término, con haber tenido la oportunidad de interactuar en el espacio offline con algunos compatriotas miembros de este tipo de agregaciones, dentro del contexto de estas tres ciudades, en los años 2012 y 2013. En este sentido, la experiencia de interacción física en el espacio local con personas, grupos (parches, combos) o asociaciones que tienen como criterio común de identificación (dentro de la propia diferenciación interna) la nacionalidad de procedencia, sirve como antecedente para la aproximación, a través de la etnografía virtual, a estas formas de agregación social-virtual.

Aun cuando inicialmente no participaba de estas agrupaciones online, más por una cuestión de resistencia contracultural al ingreso en el mundo de Facebook, la necesidad de comunicación en momentos de precariedad durante el tránsito como inmigrante motivó la inmersión en este, para mí, nuevo escenario. Por esta razón, la indagación sobre el objeto de estudio se delimita temporalmente al año 2013, momento en el cual ingresé a estos grupos, casi simultáneamente. La motivación personal para ingresar era principalmente poder estar en contacto con los compañeros colombianos en Buenos Aires y en La Plata, en donde pasaba temporalmente el periodo de búsqueda de residencia para el nuevo año de estudio, además de observar las ofertas de lugares de habitación en una y otra ciudad. La decisión de tomar esta población objetivo para mi trabajo sobre tecnologías de la información y comunicación se produce de manera simultánea, al ver la oportunidad de no solo desarrollar una aproximación teórica al concepto de comunidad virtual, como era el propósito inicial, sino de confrontar el plano conceptual en la propia experiencia compartida de pertenencia a este tipo de formas de agregación.

Es necesario igualmente señalar que para el propio planteamiento de esta indagación es fundamental la relación de la percepción y la experiencia adquirida en el paso por estas tres ciudades. Dadas sus condiciones geográficas, culturales y sociodemográficas son importantes aspectos generales a considerar: 1) el tamaño de la ciudad -desde las connotaciones de urbe superpoblada en el caso del conurbano bonaerense, pasando por la propia incorporación y búsqueda distintiva de La Plata respecto de la urbe, ciudad que junto con Bahía Blanca tendrían unas condiciones más propias de localidades intermedias-; 2) su condición jurídicopolítica frente al territorio de la nación -capital federal en el caso de Buenos Aires, capital de la provincia de Buenos Aires en el caso de La Plata-; 3) el carácter multidimensional de la vida de la urbe, respecto de las dimensiones establecidas como referentes de identificación en el caso de La Plata - universidad, burocracia-, y Bahía Blanca -estamento militar, industria- 
así como los demás imaginarios sociales que se desprenden de sus propias configuraciones identitarias.

De igual manera cabe señalar, a manera de contexto introductorio, la percepción sobre la diferente composición social de la inmigración colombiana en cada una de las ciudades en las que se desarrollan estas formas de agregación social-virtual: en ese sentido es posible observar una población significativamente más estudiantil y transitoria en la ciudad de La Plata, una población inferior en tamaño y mucho más establecida en la ciudad de Bahía Blanca -sobre todo becarios de instituciones como CONICET Y mujeres colombianas casadas en Argentina- y una población mucho mayor y heterogénea en la capital federal -estudiantes de carreras de postgrado y ahora también de grado, trabajadores formales y en la informalidad en diferentes rubros, pequeños empresarios y comerciantes-.

En el proceso de interacción dentro de la población objetivo de este trabajo, el primer acercamiento se dio con los administradores de los grupos en los que me encuentro agregado, a quienes envié una solicitud inicial en estos términos:

Buenas estimada/o compatriota:

Mi nombre es César Guauque Torres, estudiante de Doctorado en Ciencias Sociales en la Universidad Nacional de La Plata. En este momento me encuentro adelantando un trabajo de exploración sobre el uso y apropiación de la plataforma de Facebook por parte de las agrupaciones de colombianos en las ciudades de Bahía Blanca, La Plata y Buenos Aires. Mi interés es especialmente observar la relación entre el contexto de cada una de las ciudades y las posibilidades de integración como colectividad colombiana, que se dan a partir de la participación en las agrupaciones en Facebook. Para mí sería de una alta significación y valor su colaboración para efectos de señalarme sus apreciaciones sobre un muy breve temario que si tiene a bien apoyarme, le enviaré una vez tenga su respuesta. En cualquier caso agradezco de antemano su interés y respuesta.

Cordialmente,

César Guauque Torres

Estudiante UNLP

De los administradores de los diez grupos sobre los cuales busqué establecer contacto, solo recibí respuesta de cinco de ellos, a quienes envié el cuestionario a manera de entrevista. De esos cinco solo contestaron la entrevista: Andrés Anzola, administrador del grupo de colombianos en Bahía Blanca y Audrin Bermúdez Zea, del grupo Casa de Colombia.

Como una forma de acceso a los demás miembros del grupo solicité apoyo a los administradores para localizar e identificar algunas personas con una participación más activa, con la intención de poder ampliar un poco, a través de las entrevistas, el conocimiento sobre las experiencias de uso, apropiación y percepción sobre la importancia del medio para la integración de los connacionales. Sobre este último paso pude establecer contacto con un miembro de colombianos en Bahía Blanca, uno de colombianos en La Plata y tres de colombianos en Buenos Aires. En este caso la entrevista fue reformulada en 
algunos aspectos, respecto de la realizada a los administradores de las agrupaciones.

Por último, y con el propósito de interactuar un poco más dentro de la agrupación, enriquecer la información y el conocimiento a partir del acceso a la experiencia de un mayor número de miembros, tuve la oportunidad de publicar en el muro de cada una de las diez agrupaciones sobre las cuales se trabajó, la siguiente solicitud acompañada de un formato de encuesta:

Hola a todos, estimados compatriotas.

Mi nombre es César Guauque Torres, estudiante de la unLP. Estoy haciendo un trabajo sobre la importancia de las redes como Facebook para la integración de los colombianos en Argentina; solicito su colaboración y buena onda para responder una breve encuesta (cuatro preguntas) que les envío en archivo adjunto. A los que puedan les estaré inmensamente agradecido.

La respuesta a este mensaje fue bastante reducida: de las agrupaciones de colombianos en Buenos Aires, que son las que tienen un mayor número de miembros y actividad, no obtuve ninguna respuesta; de los colombianos en La Plata respondieron la encuesta dos personas. Entre el grupo de colombianos en Bahía Blanca conté con una mayor receptividad, en proporción al tamaño de la agrupación, pues seis personas respondieron la encuesta.

De acuerdo con la información obtenida en este proceso de aproximación e interacción a través de la plataforma, a continuación elaboré una relación de los testimonios (principales impresio- nes, experiencias de los participantes), y de algunos datos obtenidos mediante la observación del espacio de intertextualidad. La presentación de estos resultados se da en función del orden previsto para la caracterización de la participación: comienza con las motivaciones para el ingreso, continúa con los sentidos que asume la pertenencia a estos grupos y la descripción de los principales tipos de uso y formas de apropiación, para finalizar con la mención del vínculo entre el contexto local y las posibilidades de integración que ofrece este espacio desde la copresencia online hasta el contacto y articulación fuera de Internet.

\section{A. Las motivaciones para el ingreso a las asociaciones en Facebook de colombianos en Argentina}

En su proceso de incorporación y adaptación a una nueva vida en este país, las principales razones que tienen los colombianos para ingresar a las agrupaciones en Facebook van desde la necesidad de asistencia, apoyo o consejo sobre las condiciones de vida en Argentina; hacer los trámites de inmigración; buscar habitación o apartamento en alquiler y organizar su permanencia en los primeros días, pasando por el muy importante restablecimiento del vínculo social en su condición de migrante con sus compatriotas, hasta el denominado "rebusque" o promoción de emprendimientos de diversa índole con el fin de generar ingresos para mejorar sus condiciones de vida en la ciudad en que habitan.

Audrin Bermúdez Zea, administradora de la página Casa de Colombia (grupo cerrado), afirma 
que una vez radicada en Argentina, para hacer frente a las dificultades en materia laboral y generar algunos ingresos "fue fundamental la vinculación a redes de Facebook pues allí ofrezco diversos productos y servicios a la colectividad colombiana". En su caso, la principal motivación para ingresar a las demás agrupaciones que conoce (Colombianos en Baires, Colombianos en Argentina, Colombianos Radicados en Argentina), además de crear este grupo, fue la oferta y demanda de productos y servicios.

A su vez, Andrés Anzola, administrador del grupo de Colombianos en Bahía Blanca (grupo cerrado), considera que uno de los principales motivos para que los inmigrantes colombianos se unan a este tipo de asociaciones tiene que ver con mantener de alguna forma el contacto con su patria, compartiendo con amigos de su tierra, puesto que permiten, "tener un espacio donde estar en contacto y acceder a información de compatriotas que seguramente hayan vivido situaciones similares, por ejemplo, saber dónde hay eventos colombianos o cómo conseguir productos, hacer trámites propios de nuestra nacionalidad".

En el caso de otra de las personas entrevistadas, María Angélica Farfán, integrante de Colombianos en Argentina, Colombianos en Buenos Aires y Mujeres colombianas en Buenos Aires, la principal motivación es el sentimiento de familiaridad que une a los colombianos residentes en Argentina; en ese sentido estas asociaciones son "un buen espacio para enterarse de eventos, actividades, emprendimientos o problemas que puedan presentársele a otros compatriotas o para consultar ante cualquier eventualidad".

En el caso de colombianos en La Plata, Paula Andrea Ramírez, administradora de la agrupación en Facebook (grupo abierto), comenta que las principales motivaciones para vincularse al grupo por parte de los compatriotas recién llegados a la ciudad tienen que ver con buscar orientación de sus pares sobre cuestiones básicas de supervivencia: Iugar para vivir, trámites, dónde conseguir cosas, etc.

En relación con las personas que respondieron al formato de encuesta (ocho en total: seis de Bahía Blanca y dos de La Plata), la principal motivación para unirse a estos grupos es la integración con otros colombianos, seguida de compartir y ser solidario en su experiencia como inmigrante y, en tercer lugar, la necesidad de apoyo, asistencia o información.

\section{B. Sentidos que asume la participación en las agrupaciones}

Una vez se generan las condiciones para tener un cierto nivel de estabilidad en su experiencia como inmigrante, el uso y apropiación de la plataforma por parte de los miembros de las agrupaciones va adquiriendo otros sentidos, sin perder su connotación inicial de medio para obtener asistencia, consejo, apoyo y como espacio para la promoción de bienes y servicios. La construcción de sentido común, como colombianos inmigrantes que a pesar de la propia diferenciación interna en cuanto a la región o condición de origen comparten unos imagina- 
rios de identificación, es quizás el aspecto más importante de la interacción.

El ser colombiano es en sí mismo un significante vacío, que es poblado desde las diferentes formaciones discursivas que encarnan la diversidad regional, cultural y social -desde el nivel étnico, pasando por el ethos, las acentuaciones y temperamentos, hasta la gastronomía y las expresiones musicales-. Existen, no obstante, una serie de discursos, objetos y expresiones que en la disputa por el valor simbólico imponen su significado, al tener una mayor representatividad desde lo que los colombianos perciben que es considerado como más típico a partir de una mirada externa: la mochila arhuaca; la música vallenata y la salsa; la gastronomía "nacional" en la que se imponen las arepas, la bandeja paisa y bebidas alcohólicas como el aguardiente; el llamarnos entre nosotros "parceros" o el manifestar el estado de insuperable satisfacción con la expresión "una chimba", son todos símbolos que se unen a una serie de tópicos de autoidentificación con fundamento en valores arraigados en la tradición, como la laboriosidad y el emprendimiento: "colombiano no se vara", la religiosidad en algunos casos, el vínculo afectivo con la familia y el territorio, y un reciente nacionalismo de cuño autoritario. ${ }^{6}$

Son todas estas manifestaciones por las cuales, en el propio espacio de conectividad se expresan, ponen en cuestión y reconfiguran valores y

6 Durante el gobierno de Álvaro Uribe Vélez (2002-2010) se produjo una sobredeterminación hegemónica de formaciones discursivas, que dio lugar a una fuerte reconfiguración de los patrones de identificación bajo la exacerbación de un nacionalismo contrainsurgente, más precisamente antifariano. símbolos de identificación que permiten la realización de la comunidad virtual como comunidad de sentido que palpita y se moviliza en el propio espacio de intertextualidad generado por esta forma de sociabilidad virtual. La comunidad virtual adquiere de esta manera una materialidad espectral como presencia viva de esa intersubjetividad que construye y disputa el sentido de la "colombianidad" a partir de la agrupación, que es tan solo su modo pasivo, lo cual es particularmente importante en el caso de los grupos más grandes (COLOMBIANOS EN BUENOS AIRES, COLOMBIANOS EN BAIRES) en los cuales el anonimato y la dispersión que permite la masividad de la agrupación, en el contexto de una urbe superpoblada, favorece la espontaneidad en la expresión y la problematización de algunos temas que tratan de ser evitados en espacios más pequeños y de mayor proximidad, como la agrupación de Colombianos en Bahía Blanca, en la cual la mayoría de sus miembros se conocen entre sí.

En el aspecto que nos ocupa, las manifestaciones de orden político son particularmente importantes para observar cómo las tensiones producto de la diferenciación y polarización existente en Colombia son reproducidas en la condición de inmigrante, siendo más explícitamente planteadas en las agrupaciones más grandes, en las cuales interesa más la opinión y la movilización de la presencia virtual que la poco probable y de seguro muy incómoda posibilidad de encuentro con la presencia física del otro. A la inversa, en el grupo más pequeño se prioriza la identificación como colectivo respecto de la diversidad interna, procurando dejar a un lado escenarios de confrontación. 
La oferta y demanda de bienes y servicios es uno de los principales sentidos que adquiere la participación de los miembros dentro de la agrupación, utilización que refuerza en alguna medida el tópico de autoafirmación identitaria de los colombianos como emprendedores. Sin embargo, el medio también es usado por muchos argentinos especialmente para la oferta inmobiliaria y de servicios. Tal como comenta Audrin Bermúdez Zea: "Participo activamente ofreciendo los productos y servicios de mi emprendimiento, también respondo inquietudes de compatriotas a quienes puedo ayudarles con información y opino en temas de interés para la colectividad."

Muchas personas solo se involucran en la actividad del grupo como una manera de entretenimiento, pasando el tiempo más como merodeadores que como participantes activos. También se presentan algunos que solo observan la actividad de la agrupación desde su espacio personal y ocasionalmente ingresan para mirar algún aviso, información o comentario que les parece interesante al verlo publicado en su muro, situación que parece ser usual en quienes hacen parte de las agrupaciones más grandes, tal como señala María Angélica Farfán de acuerdo con su experiencia en el grupo Colombianos en Buenos Aires.

Andrés Anzola señala que en el caso de colombianos en Bahía Blanca, las motivaciones para participar en la agrupación tienen que ver más con propósitos organizativos, de divulgación y consulta. Tal como veremos en los datos correspondientes a la observación de la actividad del grupo, el tipo de apropiación con fines de organización de la colectividad representa más de la mitad de su movimiento. Este tipo de apropiación es la más observada, según la información aportada por los miembros de Colombianos en Bahía Blanca que contestaron la encuesta, quienes comparten contenidos acerca de actividades para realizar en la ciudad e invitan a reunirse a sus compatriotas no solo para participar en la agenda cultural, sino fundamentalmente para encuentros de carácter social: comidas, fiestas, paseos, etc.

La observación-participante desarrollada me permitió, igualmente, señalar como indicador de un mayor nivel de apropiación sobre el espacio, el planteamiento de escenarios de debate sobre el grupo. No son frecuentes los momentos de reflexividad de los miembros sobre su participación y el uso que se hace de las agrupaciones, sin embargo pude identificar y tratar de tomar posición dentro de una discusión que se presentó en el grupo Colombianos en La Plata. El cuestionamiento fue planteado por parte de un integrante que -molesto por el excesivo uso para fines de mercadeo del espacio de la agrupación, especialmente por parte de argentinos- solicitó a los administradores tener un poco más de cuidado en la verificación de las condiciones de ingreso. Algunas personas lo acusaron de propiciar formas de exclusión y otras se sumaron a su posición; en cualquier caso, se generó un espacio de autoobservación por medio del cual los miembros asumieron alguna postura frente a lo que debería ser o hacerse con el grupo. 
Extracto de la plataforma una parte del mensaje de un miembro de la comunidad, identificado como Zul Andre Salgado, que plantea la discusión:

Cuando llegué a Argentina hace casi 3 años, iniciaron las chicas de Arepas Col vendiendo un buen producto, algo que a todos nos hace falta, era fantástico, se hacían publicaciones con inquietudes que todos tenemos y sabemos, como compatriotas, alguno va a responder, apoyo todo lo que sea colombiano, pero me parece jartísimo ver cómo de cada 10 publicaciones 7 son argentinos vendiendo servicios de cualquier tipo de mierdas que entre nosotros podemos encontrar, deberíamos reconsiderar un poco sobre el nombre de la página!! Sería bueno que los administradores de la página se fijen más a la hora de permitir el ingreso de personas.

\section{Observación de los tipos de usos y formas de apropiación}

Una vez vinculado en la plataforma, agregado en los grupos y ya en ejecución de la investigación, consideré la posibilidad de desarrollar la observación sobre el espacio de intertextualidad de las agrupaciones en los seis primeros meses del año 2013, alternativa que luego solo fue viable para el caso de Colombianos en Bahía Blanca, pero que me vi obligado a replantear dada la cantidad de miembros e interacciones de las asociaciones de Colombianos en La Plata y sobre todo en Buenos Aires. Finalmente, opté por tomar como referente temporal comparativo el periodo que va del 1 de junio al 1 de julio del año 2013.
De la misma manera debo aclarar que fue preciso excluir de esta tarea los grupos más grandes como colombianos en buenos aiRes, Colombianos en baires y Colombianos Residentes en Argentina. No obstante, ello no significa que los haya dejado de lado en esta aproximación, pues sobre estas agrupaciones me fue posible realizar algunas entrevistas e intentos de practicar el muestreo, sin éxito, como mencioné anteriormente. En todo caso, pude hacer la observación también sobre algunas agrupaciones de Buenos Aires con menor volumen de información.

Como es posible verificar en el cuadro 1, el trabajo de observación comparativa de los textos publicados sobre la base de las variables propuestas tiene relevancia para establecer algunos hallazgos interesantes. En primer lugar, cómo en las agrupaciones que toman como referencia la ciudad de Buenos Aires, el principal uso es la comercialización de productos y la promoción de emprendimientos de todo tipo: comida colombiana, servicio de remises, viajes, lugares de entretenimiento, alquiler inmobiliario, bebidas alcohólicas nacionales, entre otros (en un porcentaje siempre superior al 80\%). Esta orientación es menos frecuente en el caso de Colombianos en La Plata (56.8\%) y no aparece en el caso de Colombianos en Bahía Blanca. Por el contrario, la apropiación para propósitos relacionados con la integración es mucho mayor (52.3\%) en el caso de Colombianos en Bahía Blanca, respecto de Colombianos en La Plata (5.35\%) y de las agrupaciones en Buenos Aires en donde oscila entre el 1 y el $3 \%$. 
Cuadro 1. Observación de los tipos de usos y apropiaciones de la plataforma de Facebook por parte de las agrupaciones de colombianos en Argentina ${ }^{7}$

\begin{tabular}{|c|c|c|c|c|c|c|}
\hline $\begin{array}{l}\text { Agrupaciones } \\
\text { ciudades } \\
\text { variables }\end{array}$ & $\begin{array}{l}\text { Col. en } \\
\text { La Plata }\end{array}$ & $\begin{array}{l}\text { Col. en } \\
\text { Bahía } \\
\text { Blanca }\end{array}$ & $\begin{array}{c}\text { Casa } \\
\text { de Colombia }\end{array}$ & $\begin{array}{c}\text { Col. en } \\
\text { Argentina } \\
\text { (Buenos Aires) }\end{array}$ & $\begin{array}{c}\text { Col. en Buenos } \\
\text { Aires }\end{array}$ & $\begin{array}{c}\text { Colombia en } \\
\text { Argentina }\end{array}$ \\
\hline $\begin{array}{l}\text { Integrantes a } \\
31 / 07 / 2013\end{array}$ & 1637 & 106 & 720 & 1092 & 569 & 1178 \\
\hline $\begin{array}{l}\text { Apropiación } \\
\text { apoyo, asistencia / } \\
\text { consejo. }\end{array}$ & $19823.5 \%$ & 4 19.04\% & $\begin{array}{l}5 \\
5 \%\end{array}$ & $\begin{array}{l}26 \\
3 \%\end{array}$ & $\begin{array}{l}22 \\
4.7 \%\end{array}$ & $\begin{array}{l}12 \\
1.4 \%\end{array}$ \\
\hline $\begin{array}{l}\text { Uso particular: } \\
\text { comercial, promo- } \\
\text { cional }\end{array}$ & $47856.8 \%$ & 0 & $\begin{array}{l}86 \\
86,8 \%\end{array}$ & 774 91.4\% & 414 88.65\% & $75892.8 \%$ \\
\hline $\begin{array}{l}\text { Uso con fines de } \\
\text { integración offline } \\
\text { de la asociación. }\end{array}$ & $455.35 \%$ & $\begin{array}{l}11 \\
52.3 \%\end{array}$ & $\begin{array}{l}3 \\
3 \%\end{array}$ & $\begin{array}{l}9 \\
1.06 \%\end{array}$ & $\begin{array}{l}6 \\
1.2 \%\end{array}$ & $\begin{array}{l}6 \\
0.7 \%\end{array}$ \\
\hline $\begin{array}{l}\text { Apropiación con } \\
\text { fines expresivos, } \\
\text { simbólicos. }\end{array}$ & 97 11.5\% & $\begin{array}{l}5 \\
23.8 \%\end{array}$ & $\begin{array}{l}5 \\
5 \%\end{array}$ & $\begin{array}{l}34 \\
4 \%\end{array}$ & $\begin{array}{l}19 \\
4 \%\end{array}$ & $\begin{array}{l}37 \\
4.5 \%\end{array}$ \\
\hline $\begin{array}{l}\text { Desarrollo de } \\
\text { problemas/ tensio- } \\
\text { nes inmigración. }\end{array}$ & $232.73 \%$ & $\begin{array}{l}1 \\
4.7 \%\end{array}$ & 0 & $\begin{array}{l}3 \\
0.3 \%\end{array}$ & $\begin{array}{l}6 \\
1.2 \%\end{array}$ & $\begin{array}{l}3 \\
0.3 \%\end{array}$ \\
\hline
\end{tabular}

\section{DESDE LA COMUNICABILIDAD VIRTUAL HACIA LA INTEGRACIÓN OFFLINE}

Con fundamento en lo observado hasta el momento, a partir de la interacción dentro de las agrupaciones es posible señalar que la plataforma comunicacional que proporciona Facebook, en sí misma, es solo un hábitat para estos "nativos digitales", tal como señala Roselló Reina: "El software social no es una red social, sino un instrumento que facilita la interacción entre los seres humanos. Decir que MySpace es una red

$7 \quad$ El periodo de observación de las interacciones en los grupos se realizó entre el 01/06/2013 y el 01/07/2013. Se caracterizaron los textos compartidos en las agrupaciones con base en las variables propuestas. social es como decirle agua al vaso que la contiene" (2011, pág. 5).

Sobre el espacio y los recursos técnicos que proporciona ese software se despliegan formas de agregación e interacción que tienen como imaginario constitutivo la idea de red social. Las agrupaciones que se van produciendo, favorecidas por la accesibilidad, la multipresencia de la personalidad online y la dificultad para ejercer términos de coacción formales sobre los vínculos de lealtad y el compromiso de los participantes, son formas de interconexión que en este territorio virtual pueden configurar, de acuerdo con Hands, desde enjambres espontáneos (swarms) -subredes emergentes a partir de coyunturas, pulsiones y movimientos de opinión- 
hasta redes de reconocimiento en las cuales se despliega la acción comunicativa que permite el diálogo y el consenso (citado en Burch, 2011, pág. 15).

Tal como señalé en el caso de las agrupaciones más grandes, el propio espacio de intertextualidad sobre el cual se expresan, cuestionan y reconfiguran valores y símbolos de identificación se convierte en el plano sobre el cual se produce la comunidad, espacio siempre en tensión entre la entusiasta emergencia y la normalizadora inercia. Como la cresta de la ola, "que cuando logra ser ya no es ninguna" de que nos habla Silvio Rodríguez, se presentan movimientos en los cuales se observa el ascenso y declive de la participación. En muchas ocasiones esta se despliega a partir de manifestaciones de provocación, pasa por el entrecruzamiento de posiciones y por un momento de máxima exacerbación (el cual si bien puede dar albergue a algún sentido común, nunca produce un cierre, ni siquiera precario), para desintegrarse finalmente al desembocar de nuevo en la polifónica multidimensionalidad constitutiva de las subjetividades que le dieron forma.

Para ilustrar esta afirmación tomo como ejemplo una tensión que se manifiesta en el contexto de la inmigración colombiana, en relación con el establecimiento de límites o restricciones para el ingreso de colombianos a la Argentina. Los procesos de extrañamiento y de afianzamiento de la interioridad constitutiva a partir de la otredad puesta al margen dentro de las fronteras culturales de lo social, en la mayoría de los casos agenciados desde comprensiones polici- vas de la sociedad, tienen hoy eco en una interpretación peligrosista del incremento que en los últimos cinco años ha tenido la inmigración colombiana. ${ }^{8}$ Las formas de generalización propias del populismo punitivo replican y reproducen mediáticamente la imagen del colombiano asociada a la delincuencia común y el narcotráfico, tomando como sustento la innegable existencia de un nunca bien determinado número o proporción de conductas ilícitas cometidas por personas de nuestra nacionalidad. Lamentablemente son los propios colombianos residentes en Argentina quienes hacen mayor resonancia de este tipo de dispositivos sociales de etiquetamiento, produciendo un clima hostil contra sus propios compatriotas recién llegados, y manifestándose abiertamente en favor del establecimiento de restricciones y límites jurídicos que a ellos no les fueron exigidos. En suma, la existencia efectiva del problema, magnificado eso sí por las formas de control social y criminalización jurídico-políticas han llevado al Gobierno argentino a establecer recientemente mecanismos de evaluación y contención, como el requerimiento de visado a quienes desean ingresar al país en calidad de estudiantes, requisito dentro del cual se debe acreditar la posesión de recursos suficientes para el sostenimiento económico durante el tiempo de permanencia.

8 De acuerdo con algunos estudios, en el 2008 se incrementó en más de $200 \%$ el número de solicitudes de colombianos para radicarse en el país, respecto del promedio del periodo 2002-2008. "De esta forma, se pasó de 500 radicaciones por año a 5500 en 2008, record histórico comparado con toda la década, que representa el máximo aumento de porcentaje registrado entre todas las nacionalidades" (Hernández, 2011, pág. 11). Adicionalmente, y conforme a cifras de la base Sadex sobre las radicaciones iniciadas por nacionalidad por año, se pasó de 5.442 en 2008 a 16.422 en 2012. 
Es precisamente este tema uno de los más poderosos tópicos de movilización de estas agrupaciones para su configuración y agenciamiento como comunidad virtual. Su latencia hace que sea recurrentemente puesto en cuestión generando apasionados comentarios, por lo general en favor del reforzamiento de la frontera, así como también encarnizados debates sobre los destinatarios y alcances que deben tener estas medidas. La comunicabilidad produce la comunidad virtual en el movimiento del grupo que en el acto de compartir construye sentido. En este caso, como afirma Winocur, "lo comunitario ya no alude a los fuertes lazos que se establecen a partir del compromiso impuesto por la tradición, los mayores o la necesidad de supervivencia, sino a los gustos y preferencias compartidas y escogidas libre e individualmente" (2005, pág. 92).

El siguiente es solo uno de los muchos ejemplos de comentarios expresados en desarrollo de esa tensión. Jorge Luis Morales comparte en la agrupación COLOMBIANOS EN BUENOS AIRES un titular del diario Clarín: "Crimen de sicarios: le pegan cuatro tiros a un colombiano", manifestando de paso su preocupación por las consecuencias que en términos de estigmatización y discriminación social puede generar este tipo de agendas informativas del principal medio impreso de Argentina.

Para seguir caracterizando esta clase de comunidades en términos de Winocur, observo cómo este tipo de nucleamiento en particular tiene una connotación claramente sectorizada. Tal como pudimos observar en los testimonios de los miembros de los diferentes grupos, se toma como eje aglutinador la condición de inmigrante y la nacionalidad de origen, entre otras cosas, para "la gestión u obtención de servicios, la ayuda mutua, el apoyo psicológico, la búsqueda de reconocimiento o el deseo de trascender a través de los medios más allá de las fronteras domésticas e institucionales donde se mueven habitualmente" (Winocur, 2005, pág. 97). Más específicamente, en el contexto de las grandes ciudades, las agrupaciones virtuales permiten restablecer, así sea de manera precaria, vínculos de afinidad y solidaridad que en muchos casos sirven como apoyo especialmente cuando se presentan situaciones extremas, tal como pudimos constatar en la reacción y respuesta para la asistencia humanitaria no solo con los compatriotas, sino en general con la población afectada por las inundaciones de principios del mes de abril de 2013 en La Plata y Buenos Aires.

No obstante, en el caso de las agrupaciones más grandes, es claro cómo la principal función que cumplen estas formas de sociabilidad virtual se encuentran vinculadas a los procesos de construcción y reconstrucción de las identidades (Winocur, 2005, pág. 97). La disputa por la identidad, como capital simbólico del grupo, entre las diferentes formaciones sociales-discursivas expresadas desde las diversas posiciones e intereses, pone en cuestión y reconfigura valores y símbolos como parámetros de identificación, otorgando un reconocimiento precario a determinadas formas de ser y de hacer. Este reconocimiento difícilmente puede pretender un cierre de sentido al quedar expuesto a su reformulación permanente. 
Con fundamento en esta caracterización, es posible afirmar la existencia de la comunidad virtual en la configuración de la presencia espectral de esa comunicabilidad. Sin embargo, la capacidad de esta comunidad para trascender su realidad virtual y proyectarse hacia la sociabilidad fuera de Internet debe entrar a ser analizada, señalando en este punto cómo incide el contexto de las ciudades estudiadas en las posibilidades de integración offline de los miembros de la agrupación.

En primer término, las dimensiones de la población de inmigrantes colombianos, sobre todo en Buenos Aires y La Plata, hace que la apropiación de la agrupación para desarrollar formas de integración de los participantes en el mundo offline funcione solo de manera relativa y limitada. En efecto, se producen iniciativas para compartir espacios de entretenimiento, vida social, farra o rumba en términos colombianos, invitaciones que en la mayoría de los casos son realizadas por los propietarios de los establecimientos en donde tienen lugar los encuentros. En muy pocos casos se usa este medio para promover reuniones de otro tipo. Los muy numerosos combos o parches políticos de colombianos presentes en estas ciudades en pocas ocasiones hacen uso de estos espacios para sus convocatorias, en mi modo de ver, para evitar escenarios de confrontación. ${ }^{9}$ Mucho menos viable parece ser

9 Cabe recordar en ese sentido la reciente visita de la exsenadora Piedad Córdoba a Argentina, y su llegada a la Universidad Nacional de La Plata para dar una conferencia sobre el conflicto armado en Colombia y para recibir un reconocimiento por parte de las directivas de la Universidad. El evento fue publicitado en la página de la agrupación Colombianos en La Plata, en donde se puso en evidencia la extrema polarización de la sociedad colombiana, que es reproducida en la condición de inmigrante. la posibilidad de convergencia para la organización como colectividad colombiana. En este contexto, tales agrupaciones, además de reproducir las lógicas de diferenciación y sectarismo que vienen de la sociedad colombiana, se inscriben dentro de un escenario heterogéneo y fragmentado por las mismas condiciones del medio receptor, haciendo muy difícil cualquier intento de articulación entre las diferencias.

Así parece desprenderse de los testimonios de las compatriotas residentes en Buenos Aires, quienes como en el caso de María Angélica Farfán no ha usado la agrupación con ese propósito, y Audrin Bermúdez que lo ha hecho solo de manera limitada. Las dos reconocen, sin embargo, que algunos compatriotas lo utilizan para hacer amigos y con fines sociales de esparcimiento. Para Audrin, "mi integración con otros colombianos en general empieza por temas de mi emprendimiento y de allí he podido rescatar un par de personas con quienes hemos construido un vínculo más cercano a la amistad."

En el caso de La Plata, las características propias de la población de colombianos residentes en esta ciudad, en su inmensa mayoría estudiantes, influyen mucho más que el contexto de la ciudad para las posibilidades de integración de los miembros de la agrupación en el espacio offline. Paula Andrea Ramírez, administradora del grupo, plantea en ese sentido:

La mayoría de los colombianos pertenecientes al face son estudiantes y por tanto están como habitantes transitorios de este país, con lo cual no tienen interés de pertenecer a una colectividad, me parece. Estudian en Argentina pero 
siguen "viviendo" en Colombia. En lo personal no me interesa hacer colectividad colombiana porque quiero estar integrada a la Argentina.

Para los dos miembros de colombianos en La Plata que contestaron la encuesta, la incidencia de la agrupación para la integración de la colectividad colombiana es mínima, en la medida en que permite estar informado sobre algunos espacios pero sin motivar a la participación.

En cuanto a la ciudad de Bahía Blanca, las posibilidades de concertación y convergencia de los participantes a través de su agrupación en Facebook son mucho mayores, en buena medida por la proximidad y cercanía que permite la copresencia de un menor número de colombianos en una localidad más pequeña. A ello se suman las condiciones de estabilidad que han permitido una mejor incorporación de esta población dentro de la comunidad y la cultura bahiense. Se puede verificar, en este caso, la existencia de una formación social con vínculos afectivos concretos, que aprovecha las posibilidades que ofrece la plataforma para fortalecerse.

\section{Como comenta Amparo López:}

Bahía Blanca es una ciudad pequeña donde es fácil y rápido moverse, y eso facilita que los miembros del grupo de Facebook nos veamos con frecuencia, organicemos eventos sociales o de la colectividad, e incluso entre nosotros hay pequeños grupos que son muy unidos y que se reúnen con mucha frecuencia; de mi parte veo a muchos como mi familia aquí y sé que no soy la única.
La comunidad virtual es aquí un fenómeno posterior, que tiene un carácter de medio para un proceso de agregación social con otros móviles. La producción de lo común como parte de la propia experiencia de tener lugar, como singularidad-colectiva, permite establecer que la representación de este ser colectivo en el espacio virtual no se trata meramente de una colección de contactos, o una práctica de agregación de una presencia virtual escindida de su encuentro y vinculación social en el espacio físico.

Un elemento fundamental para el fluido tránsito e intermediación entre la presencia física de la agrupación y su presencia virtual, en el caso de Colombianos en Bahía Blanca, es la incorporación como colectivo dentro de las actividades culturales, eventos conmemorativos, de beneficencia y festividades de la localidad. En este ejemplo hay una clara referencia a la participación de la colectividad en un evento de la municipalidad, con ocasión del cual se despliega una actividad de integración desde la misma reunión para la selección del baile, pasando por los ensayos, hasta la presentación misma. El texto compartido por el administrador de la agrupación colombianos en Bahía Blanca, señala:

Están cordialmente invitados a la reunión de la colectividad, que se efectuará el día Domingo 8 DE JUNIO (2013) A LAS 20:00 en la casa de Katy (Pueyrredón 55).

Temas a tratar

- baile en teatro municipal

- convocatoria para el baile 
- curso de bromatología

saludos

Andrés Anzola señala que el uso de la agrupación en Facebook, "a nivel de la colectividad permite organizar los eventos y conocer nuevos compatriotas, los cuales en la mayoría de los casos se convierten en amigos". Así mismo, los miembros del grupo que respondieron la encuesta coinciden en señalar que su relación con los demás colombianos en la ciudad ha sido de fraternización y articulación como colectividad colombiana, para lo cual ha sido importante la agrupación, en tanto permite coordinar encuentros y reuniones para integrarse.

La hipótesis planteada en el sentido de contrastar dos modos de configuración y existencia de la comunidad virtual: una que toma a la comunicación como un fin en sí mismo de la agrupación, a través de una forma de sociabilidad débil, y otra que tiene en la interacción online solo un medio para profundizar los vínculos de una agrupación offline existente fundada en vínculos de sociabilidad fuertes, puede entrar a cuestionarse en la medida en que, en primer lugar, se reinterpreten las formas de sociabilidad de acuerdo con las nuevas dimensiones que adquiere la acción y la interacción social en el espacio virtual, lo que como veremos demanda un esfuerzo por superar los marcos rígidos de la epistemología moderna, y, en segundo término, por el señalamiento de su imposibilidad misma, en el caso de comunidades cuya existencia y proyección no pasan por su presencia en el territorio virtual.
La comunidad virtual propiamente dicha estaría más próxima en el caso de agrupaciones como la de Colombianos en Buenos Aires, en donde la imposibilidad de la presencia en el territorio físico de una colectividad colombiana permite la ocupación y apropiación del territorio virtual por parte de la comunicabilidad de sus miembros, que en el caso de la agrupación Colombianos en Bahía Blanca, espacio que tiene más un carácter de instrumento, sin llegar nunca a concretar una entidad independiente de la copresencia e integración de sus miembros en el mundo offline.

\section{A MODO DE CONCLUSIÓN: UNA CRÍTICA}

En relación con la comprensión del concepto de comunidad virtual, uno de los principales problemas es la forma de abordaje, todavía muy fijada desde una fundamentación epistemológica moderna que no se compadece con los profundos cambios que la cada vez mayor presencia del ser en el espacio virtual tiene para la construcción del conocimiento.

En particular, los cuestionamientos que se hacen desde las nociones de autenticidad e identidad se fundamentan en una concepción estática y esencialista, por la cual la identidad se asume como un sustrato que de entrada condiciona la presencia online de los participantes en este tipo de formas de agregación, sin tomar en cuenta el espacio virtual mismo, como lugar en el cual se producen representaciones e imaginarios generadores de procesos de identificación. 
En ese sentido, desarrollos más prospectivos sobre la comprensión de la comunidad como el que realiza Agamben en su obra La comunidad que viene, nos permiten distanciar el concepto de comunidad de la política de las identidades. La comunidad que viene es la comunidad de las singularidades universales, de un ser tal que sea cual sea importa, punto en el que toma distancia de la política del reconocimiento de la diferencia propia del comunitarismo, en el cual se presenta una fuerte reivindicación de la identidad y en algunos casos de la tradición entendida como esencia.

Para Agamben (1996), la comunidad que viene no se constituye a partir de una esencia compartida de las singularidades; este sustrato identitario no permite fundar la comunidad, el tener lugar de la singularidad no la une con otras en la esencia o en una condición de pertenencia (ser blanco, comunista, indígena etc.), por el contrario, las dispersa en la existencia de lo múltiple. Lo común se construye sobre la base de la íntima exterioridad del ser dicho, sobre lo que expresamos en la producción de la inteligencia colectiva.

El ethos de la comunidad se da en la indiferencia de lo común y lo propio (comunidad inesencial), en la vida de una sociabilidad y una copertenencia, que son tan solo la expresión de una comunicabilidad en la que no se antepone un sentido de identidad o pertenencia, sino que se trata, más bien, de construir una forma de vida que en la propia exposición del ser dicho y en la práctica de entrecruzamiento y disputa de las polifónicas y multidimensionales subjetividades pueda expresar, cuestionar y replantear sus parámetros de identificación. En esta comunidad no es que no exista la identidad y la pertenencia, es que esa identidad y esa pertenencia no importan para formar parte de la comunidad.

Lejos del odioso cuestionamiento de Hine (2004) hacia quienes despectivamente denomina futurólogos, es preciso reivindicar el valor productivo de la teoría crítica que no se limita simplemente a desarrollar una descripción biologicista de la realidad, sino que debe contribuir en su producción procurando, por lo menos, que el conocimiento social funcione de otra forma. En este caso, y procurando aproximarnos hacia una fundamentación un poco más empírica, se pudo, no obstante, identificar algunos elementos de la comunidad que viene, en la naturaleza inesencial del sentido siempre frágilmente construido y en disputa en la comunicabilidad de esas singularidades que se ponen en juego no por lo que son, sino en su ser dicho: nunca podría establecerse, por ejemplo, algún tipo de acuerdo sobre lo que significa ser colombiano para los miembros de estas comunidades virtuales.

Una comunidad inesencial, constituida de múltiples formas como pura emergencia de esas esferas públicas en diáspora, rizomáticas. Presencia de la comunicabilidad como experiencia virtual que en su poder ser ya es; potencia materializada como espectro en el territorio virtual a la espera de su adecuada encarnación en el acto; pertenencia de las subjetividades desterritorializadas que se reterritorializan en su experiencia compartida. 
Su potencial subversivo se encuentra precisamente en su desfijación (en el erosionar permanente de la precaria certidumbre del sentido cada vez menos reductor de la complejidad), en su pura emergencialidad que las hace simultáneamente impredecibles e incapturables, en fuga de la anticipación y categorización, pero también efímeras, como la cresta de la ola. Es este precisamente su límite pero también su potencia; la búsqueda de su no lugar constitutivo las hace permanecer como una amenaza, como ese espectro que recorre el mundo trabajosamente constituido por el saber poder.

A mi modo de ver es la propia formulación epistemológica, expresa y proclamadamente moderna, desde la cual se despliega la argumentación de Hine (2004), la que le impide entender la configuración intersticial, límbica, de estas prácticas humanas en el umbral de indiferenciación entre su presencia virtual y física. La cuadratura limitante de su aproximación compartimentalizadora moderna la lleva a mirar más la oposición y superposición que la imbricación e indistinción recíprocamente constitutiva de estas formas.

La naturaleza desterritorializada del ciberespacio nos conmina a desfijarnos de los imaginarios propios de la localización física, sin embargo la coexistencia simultánea de estos cibercuerpos en el mundo offline lleva a desarrollar un proceso de reterritorialización, por el cual se imbrican los entramados de significaciones construidos a través de la presencia en uno y otro espacio. Todavía hoy la existencia no puede desvincularse del cuerpo y sus necesidades, pero cada vez más las necesidades y disciplinas sobre el cuerpo se encuentran mediadas por imperativos procedentes del mundo de la materialidad espectral; el consumo vinculado a una economía global, la cultura como un fenómeno transnacional, los símbolos y valores socialmente vinculantes cada vez más dependientes de las redes virtuales.

\section{Referencias}

1. Agamben, G. (1997). Homo sacer: el poder soberano y la nuda vida. Valencia: Pre-textos.

2. Agamben, G. (1996). La comunidad que viene. Valencia: Pre-Textos.

3. Appadurai, A. (2001). La modernidad desbordada: dimensiones culturales de la globalización. Montevideo: Ediciones Trilce.

4. Ayora Díaz, S. I. (2002). Re/creaciones de la comunidad. Espacios translocales en la globalización. Recuperado de: http:// www.bioetica.org/bioetica/ensciones 20. htm

5. Bauman, Z. (2001). En busca de la política. Buenos Aires: Fondo de Cultura Económica.

6. Burch, S. (2011). Activismo y comunicación digital. América Latina en movimiento, (463).

7. Castells, M. (2009). Comunicación y poder. Madrid: Alianza Editorial. 
8. Dirección Nacional de Migraciones. Argentina. (2012). Síntesis estadística de radicaciones. Informe del periodo enero-diciembre 2012. Recuperado de: http://www. migraciones.gov.ar/pdf_varios/estadisticas/Sintesis\%20Radicaciones\%20enerodiciembre\%202012.pdf

9. García, M. y Uscátegui, A. (Mayo 19-20 de 2011). Redes sociales virtuales. Más allá de la mediación tecnológica. Ponencia presentada en la V Conferencia acorn-redecom. Lima. Obtenido de acorn.redecom: http:// www.acorn-redecom.org/papers/2011Gil_ Espanol.pdf

10. Hardt, M. y Negri, A. (2000). Imperio. Cambridge, Massachussets: Harvard University Press. Obtenido de chilevive: http://www. chilevive.cl

11. Hernández Rodríguez, C. M. (2011). Colombianos en Argentina. Memoria del conflicto armado y experiencia migratoria. Revista de Estudios Sociales Nueva Época, 8, 189201.

12. Hine, C. (2000). Etnografía virtual. Barcelona: UOC.
13. Macintyre, A. (1987). Tras la virtud. Barcelona: Crítica.

14. Roselló, R. (2011). Hoy lo real es el mundo físico y el mundo virtual. América Latina en movimiento, (463).

15. Rheingold, H. (1993). The Virtual Community: Homesteading on the Electronic Frontier. Cambridge, Massachusetts: The Mit Press.

16. Sádaba, I. (2011). Redes sociales, redes alternativas. América Latina en movimiento, (463).

17. Valderrama, C. (2008). Movimientos sociales: tic y prácticas políticas. Nómadas, (28), 94-101.

18. Winocur, R. (2005). Posibilidades y limitaciones de la participación en las comunidades y redes virtuales. Estudios de Comunicación y Política, (14), 85-100.

19. Woolgar, S. (1996). Technologies as cultural artefacts. En W. Dutton (Ed.), Information and Communication Technologies: Visions and Realities (págs. 8-102). Oxford: Oxford University Press. 